\title{
Dependence of Structural and Magnetic Properties on Annealing Times in Co-precipitated Cobalt Ferrite Nanoparticles
}

\author{
Budi Purnama $^{1 *}$, Rafika Rahmawati ${ }^{1}$, Agung Tri Wijayanta ${ }^{2}$, and Suharyana ${ }^{1}$ \\ ${ }^{1}$ Department of Physics, Faculty of Math and Natural Sciences Sebelas Maret University Jl. Ir. Sutami $36 \mathrm{~A}$ \\ Kentingan Surakarta Indonesia 57126 \\ ${ }^{2}$ Department of Mechanical Engineering, Faculty of Engineering Sebelas Maret University Jl. Ir. Sutami $36 \mathrm{~A}$ \\ Kentingan Surakarta Indonesia 57126
}

(Received 19 June 2015, Received in final form 3 August 2015, Accepted 5 August 2015)

\begin{abstract}
Modifications in the structural and magnetic properties of co-precipitated cobalt ferrite nanoparticles can be accomplished by varying the annealing time periods during the synthetic process. Experimental results show that high-purity cobalt ferrite nanoparticles are obtained using a co-precipitation process. The dependence of the crystallite sizes on the annealing time was successfully demonstrated using XRD and SEM. Finally, vibrating sample magnetometer analyses show that the magnetic properties of the cobalt ferrite nanoparticles depend on their relative particle sizes.
\end{abstract}

Keywords : cobalt ferrite, co-precipitation, nanoparticles, time annealing

\section{Introduction}

In the past decade, magnetic nanoparticles especially cobalt-ferrite-based materials have drawn substantial attention due to their attractive magnetic properties. Cobalt ferrite nanoparticles exhibit a large magnetocrystalline anisotropy and/or surface anisotropy that is based on their coercivity or reduced remanence [1]. Due to the excellent mechanical and chemical stability of cobalt ferrite nanoparticles [2], these materials can also be integrated into information technology applications (i.e. high density recording media) [3] and medical applications as a contrast agent for magnetic resonance imaging [4] or as a tool for early cancer diagnosis and targeted therapy procedures [5].

Based on past experimental observations, it is obvious that the particle sizes of the cobalt ferrite nanoparticles influence their magnetic properties and super paramagnetic blocking temperatures $\left(T_{\mathrm{B}}\right)$. Both of these properties are essential in recording devices and biomedical applications $[3,6]$. It is well known that different synthetic procedures can result in varying nanoparticle sizes [7, 8]. A preferred

CThe Korean Magnetics Society. All rights reserved.

*Corresponding author: Tel: +62-271-669017

Fax: +62-271-669017, e-mail: bpurnama@mipa.uns.ac.id method for the synthesis of cobalt ferrite magnetic nanoparticles involves a co-precipitation process. Some papers have also reported that an additional heating step modifies the particle sizes [9-11]. This procedure could also potentially reduce the required temperature levels for the annealing process. However, little attention has been placed on the influence of time during the annealing process, which can subsequently influence the characteristics of the resulting cobalt ferrite nanoparticles.

In this study, a co-precipitation procedure was used to synthesize the cobalt ferrite nanoparticles. Modifications of the annealing times can selectively produce nanoparticles with varying sizes. The resulting cobalt ferrite nanoparticles were characterized using TG/DTA, FTIR, X-ray diffraction, SEM and a vibrating sample magnetometer.

\section{Experiment}

The synthesis and evaluation of cobalt ferrite nanoparticles involves preparing the precursor, annealing the resulting material and characterizing the final product. All the experimental procedures were performed under atmospheric conditions. $200 \mathrm{~mL}$ of $0.001 \mathrm{M} \mathrm{Co}\left(\mathrm{NO}_{3}\right)_{2} \cdot 6 \mathrm{H}_{2} \mathrm{O}$ and $0.002 \mathrm{M} \mathrm{Fe}\left(\mathrm{NO}_{3}\right)_{3} \cdot 9 \mathrm{H}_{2} \mathrm{O}$ were first added dropwise to $100 \mathrm{ml}$ of $4.8 \mathrm{M} \mathrm{NaOH}$. During the titration process, the $\mathrm{NaOH}$ solution was stirred at $1000 \mathrm{rpm}$ for $1 \mathrm{~h}$ at a 
regulated temperature of $95^{\circ} \mathrm{C}$. The solution was then continually stirred for $2 \mathrm{~h}$ until the precursor gel was formed. The product was cooled to room temperature and repeatedly washed with distilled water and acetone until a clean material was obtained. The sample was then dried overnight in an oven at about $95^{\circ} \mathrm{C}$. The second step involves annealing the material at a temperature of $600^{\circ} \mathrm{C}$ under atmospheric conditions for 2, 6 and $11 \mathrm{~h}$. The crystalline structures of the final samples were characterized by X-ray diffraction with a Bruker D8 Advance system. FTIR was used to confirm that the final materials all contained hydrocarbon compounds. The sizes of the magnetic nanoparticles were estimated based on SEM images, and the magnetic properties were evaluated using a vibrating sample magnetometer (VSM).

\section{Experimental Results}

Fig. 1 shows the TG/DTA profile of the co-precipitated cobalt ferrite nanoparticles. The three plateaus in the TG are indicative of the changes in the sample mass. A mass loss of $-0.78 \%$ is observed in the first plateau from $62.8^{\circ} \mathrm{C}$ to $135.4^{\circ} \mathrm{C}$. This plateau is considered to be hydrocarbon waste since it corresponds to an enthalpy change of $-429.24 \mathrm{~J} / \mathrm{g}$ in the DTA curve. The second plateau gives a relative mass loss of $0.36 \%$ and an associated enthalpy change of $-94.15 \mathrm{~J} / \mathrm{g}$ from $616.3^{\circ} \mathrm{C}$ to $673.2^{\circ} \mathrm{C}$. The mass loss for the second plateau is reduced by $0.42 \%(=0.78-0.36)$ when it is compared with the mass loss of the first plateau. This is most likely attributed to a dislocation of the nanoparticles towards a more stable configuration. A final plateau is observed from $804.2^{\circ} \mathrm{C}$ to $834.2^{\circ} \mathrm{C}$, where the samples experienced a $0.44 \%$ mass loss that is relatively consistent with the mass loss of the second plateau. In the case of crystal-

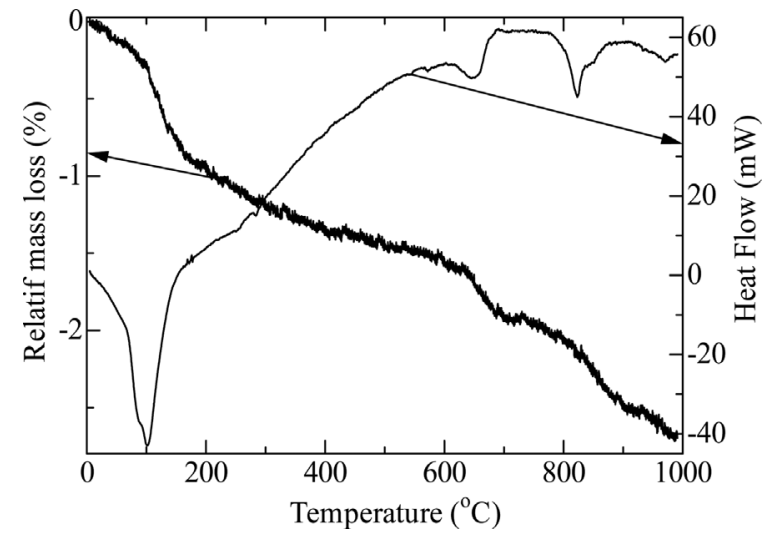

Fig. 1. Typical TG/DTA results for cobalt ferrite nanoparticles.

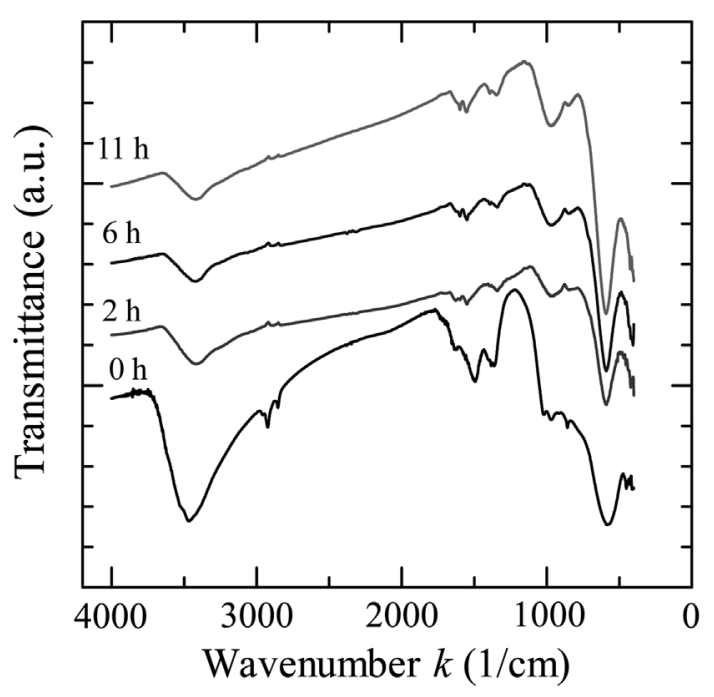

Fig. 2. The modified FTIR curve of cobalt ferrite at different annealing time periods i.e. $0,2,6$ and $11 \mathrm{~h}$.

lization, it is expected that an increase in the heating temperature beyond $616.3^{\circ} \mathrm{C}$ can facilitate the growth of cobalt ferrite crystals. When the high temperature keep for a long time, it is also the decrease of both lattice strain and defects in nano-particles cobalt ferrite. Moreover, coalescence of crystallites following increasing the average crystal size may accur [12].

Fig. 2 shows a typical FTIR curve for the cobalt ferrite nanoparticles, which were produced using the coprecipitation method and varying annealing time periods of $0,2,6$ and $11 \mathrm{~h}$ at a temperature of $600^{\circ} \mathrm{C}$. In a coprecipitated sample that has not been annealed $(0 \mathrm{~h})$, a broader absorption peak that is associated with the $\mathrm{O}-\mathrm{H}$ bond appears at a wavenumber of $3400 \mathrm{~cm}^{-1}$. In contrast, the metal-oxide bonds of cobalt ferrite appear as an absorption peak around $590 \mathrm{~cm}^{-1}$ in the FTIR spectrum. The experimental peak obtained at $575 \mathrm{~cm}^{-1}$, which was calculated using the Waldron method to give a normal spinel structure, is substantially smaller than the theoretical value. It can also be observed from Fig. 2 that the magnitude of the $\mathrm{O}-\mathrm{H}$ peak decreases as the annealing time increases. In contrast, the cobalt ferrite peak clearly increases with the annealing time, which translates into an increasing ionic strength that gives strongly dipolar crystals [13].

The X-ray diffraction patterns for the cobalt ferrite nanoparticles are depicted in Fig. 3. The samples were annealed at a temperature of $600^{\circ} \mathrm{C}$ under atmospheric conditions for 2, 6 and $11 \mathrm{~h}$. It can be clearly observed that the strongest peak occurs at an angle of $2 \theta=35.73^{\circ}$ and that this peak increases with the annealing time. These results agree reasonably with the experimental 


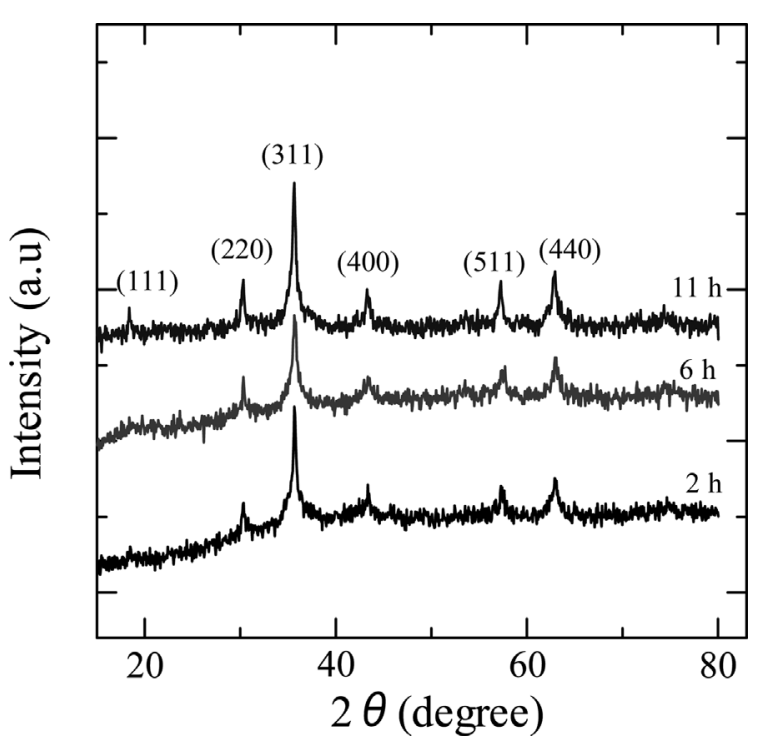

Fig. 3. X-ray diffraction patterns for cobalt ferrite nanoparticles at three annealing times of 2,6 and $11 \mathrm{~h}$ at $600^{\circ} \mathrm{C}$.

results reported by Maaz et al. There is an absence of other characteristic peaks within the XRD spectrum that indicate the presence of oxides or other impurities in the sample. Therefore, it is safe to conclude that high-purity cobalt ferrite nanoparticles were successfully produced using a co-precipitation and a low-temperature annealing process. It can also be observed that a longer annealing time decreases the background intensity in the XRD spectra. After an annealing time of $11 \mathrm{~h}$, the background intensity seems to flatten from angle $2 \theta=15^{\circ}$ to $80^{\circ}$. In the other two cases, the background intensity is slightly lower at a smaller angle rather than a larger angle. Using the strongest XRD peak and the Scherer formula, crystallite sizes of 21.4, 21.6 and $21.7 \mathrm{~nm}$ were obtained for the cobalt ferrite nanoparticles, which correspond to annealing times of 2, 6 and $11 \mathrm{~h}$, respectively.

Fig. 4 shows the SEM image of the cobalt ferrite nanoparticles with a $150,000 \times$ magnification at annealing time periods of 2 and $11 \mathrm{~h}$. The particle distribution is indicated by a small circle, which is the typical observational expectation for cobalt ferrite nanoparticles. With an annealing time of $11 \mathrm{~h}$, it seems that the small particles diffuse relative to the other particles to create bigger particles. These results are consistent with our expectations in the analysis of TG/DTA. At the beginning of the annealing process, the nano particle moving closer to one another so that interact to form a grain boundary. With increasing time, the grain boundary grew and eventually two or more nano particles merge into one larger particle size.

In contrast, an annealing time of $2 \mathrm{~h}$, affords a particle distribution that shows a distinct separation between the smaller and larger sizes. To confirm this observation, several particles that range from the smaller to the larger sizes were randomly measured for the entire area. Afterwards, the collected data was plotted on the basis of the particle size and the probability of occurrence for the varying particle sizes in Fig. 5 to afford a Gaussian-like curve. The crystallite size, which was calculated using the Scherer formula, was within the range of the Gaussian curve. The range for the cobalt ferrite particle size was 12.5 to $75.0 \mathrm{~nm}$ in the SEM image, which means that the range substantially broadens with a shorter annealing time of $2 \mathrm{~h}$. In contrast, an annealing time of $11 \mathrm{~h}$ affords a narrower particle size distribution range of only 25.0 to $65 \mathrm{~nm}$. Therefore, it can be concluded that the annealing time affects the particle size distribution of the cobalt ferrite nanoparticles.
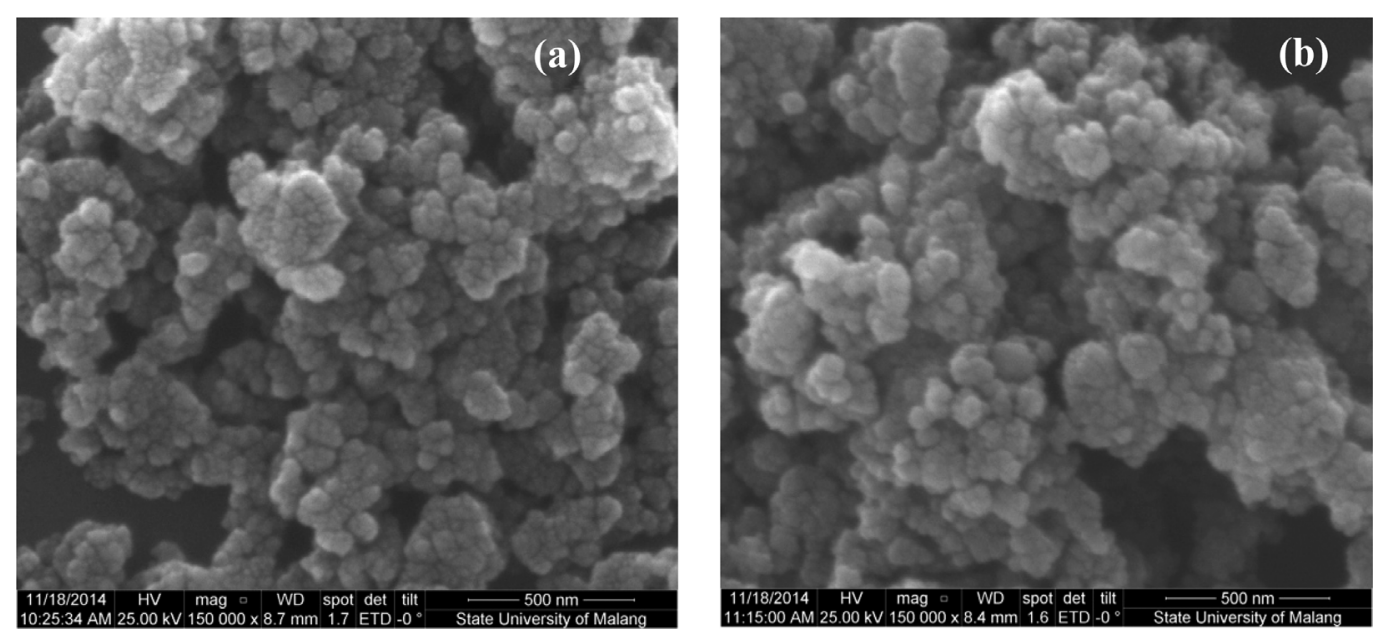

Fig. 4. Typical SEM images for cobalt ferrite after annealing at $600^{\circ} \mathrm{C}$ with two different time durations of (a) $2 \mathrm{~h}$ and (b) $11 \mathrm{~h}$. 


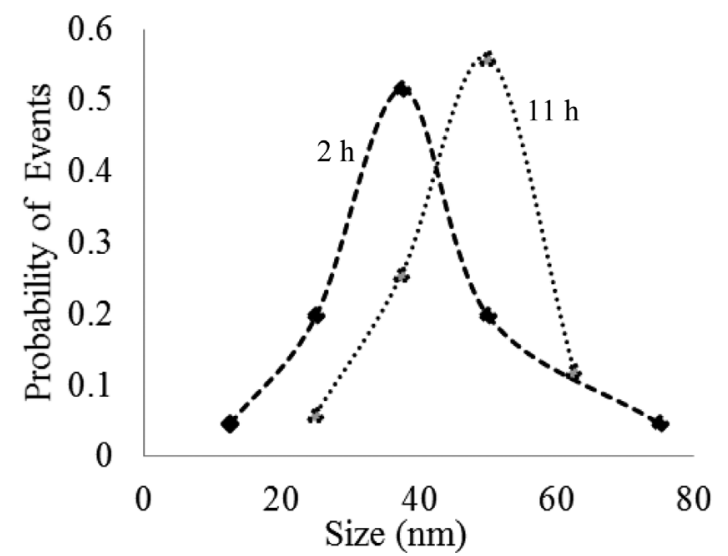

Fig. 5. Cobalt ferrite particle size distributions after two different annealing time periods of 2 and $11 \mathrm{~h}$ at $600^{\circ} \mathrm{C}$.

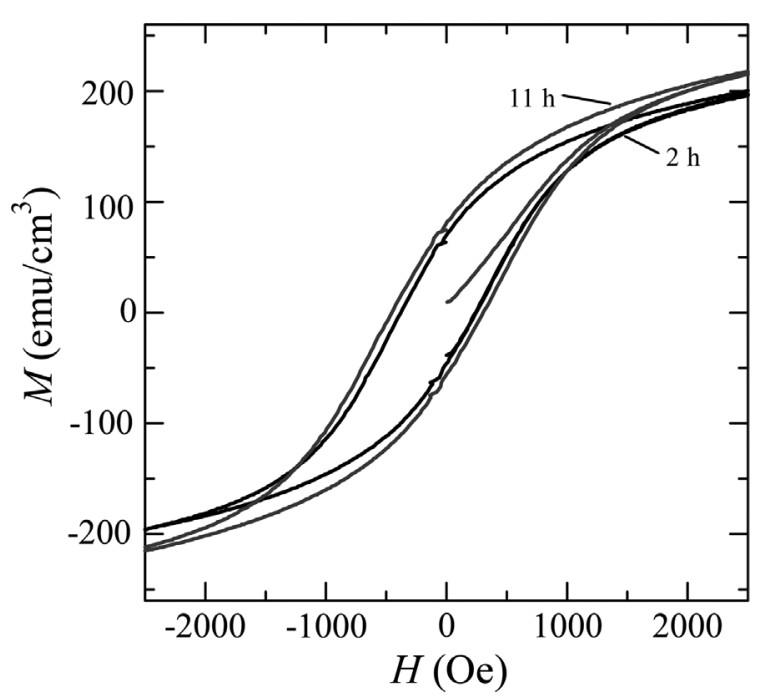

Fig. 6. A typical hysteresis curve for cobalt ferrite nanoparticles at two different annealing time periods of 2 and $11 \mathrm{~h}$.

VSM was employed to evaluate the dependence of the cobalt ferrite nanoparticle sizes on their observed magnetic properties. Fig. 6 shows the hysteresis curve for cobalt ferrite nanoparticle samples at two different annealing time periods of 2 and $11 \mathrm{~h}$. There is no difference between the squareness factors of both samples. The sample with a shorter time annealing of $2 \mathrm{~h}$ had a saturation magnetization $\left(M_{\mathrm{S}}\right)$ value of $121.3 \mathrm{emu} / \mathrm{cm}^{3}$. In contrast, a sample with a longer annealing time of $11 \mathrm{~h}$ had an $M_{\mathrm{S}}$ value of $138.3 \mathrm{emu} / \mathrm{cm}^{3}$. Coercive fields of 311 and 378 Oe were applied to the samples with varying annealing times of 2 and $11 \mathrm{~h}$, respectively. The experimental results confirmed that the magnetic property of the cobalt ferrite nanoparticles is dependent on the modified crystallite sizes.

\section{Conclusion}

The dependence of the particle sizes on the annealing time in co-precipitated magnetic cobalt ferrite nanoparticles was investigated. Experimental results show that high-purity cobalt ferrite nanoparticles can be obtained following a co-precipitation procedure. The dependence of the crystallite sizes on the annealing time was successfully proven using XRD and SEM. VSM analyses also show that the magnetic properties of the cobalt ferrite nanoparticles depend on their relative particle sizes.

\section{Acknowledgments}

This study was financially supported by the DIPA Universitas Sebelas Maret in the Republic of Indonesia (Penelitian Unggulan Perguruan Tinggi Contract No 351/ UN27.11/PN/2014).

\section{References}

[1] P. Jeppson, R. Sailer, E. Jarabek, J. Sandstrom, B. Anderson, M. Bremer, D. G. Grier, D. L. Schulz, A. N. Caruso, S. A. Payne, P. Eames, M. Tondra, H. He and D. B. Chrisey, J. Appl. Phys. 91, 114324 (2006).

[2] Y. Qu, H. Yang, N. Yang, Y. Fan, H. Zhu and G. Zou, Material Lett. 60, 3548 (2006).

[3] K. Maaz, A. Mumtaz, S. K. Hasanain, and A. Ceylan, JMMM. 308, 289 (2007).

[4] T. Ahmad, H. Bae, Y. Iqbal, I. Rhee, S. Hong, Y. Chang, J. Lee and D. Sohn, JMMM. 381, 151 (2015).

[5] V. Pasukoniene, A. Mlynska, S. Steponkiene, V. Poderys, M. Matulionyte, V. Karabanovas, U. Statkute, R. Purviniene, J. A. Krasko, A. Jagminas, M. Kurtinaitiene, M. Strioga and R. Rotomskis, Medicina. 50, 237 (2014).

[6] M. Ravichandran, G. Oza, S. Velumani, J. T. Ramirez, F. Garcia-Sierra, N. B. Andrade, M. A. Garza-Navarro, D. I. Garcia-Gutierrez and R. Asomoza, Material Lett. 135, 67 (2014).

[7] M. Houshiar, F. Zebhi, Z. J. Razi, A. Alidoust and Z. Askari, JMMM. 371, 43 (2014).

[8] K. Sinko, E. Manek, A. Meiszterics, K. Havancsa'k, U. Vainio and H. Peterlik, J. Nanopart Res. 14, 894 (2012).

[9] R. Ianos, Materials Lett. 135, 24 (2014).

[10] S. Laurent, D. Forge, M. Port, A. Roch, C. Robic, L. V. Elst and R. N. Muller, Chem. Rev. 108, 2064 (2010).

[11] K. K. Mohaideen and P. A. Joy, JMMM. 371, 121 (2014).

[12] T. P. Raming, A. J. A. Winnubst, C. M. van Kats and A. P. Philipse, J. Colloid Interface Sci. 249, 346 (2002).

[13] R. D. Waldron, Phys. Rev. 99, 1727 (1955). 